\title{
Effects of Experimental Hyperlipidemia on the Pharmacokinetics of Tadalafil in Rats
}

\author{
Joo Hyun Lee ${ }^{1}$, Ju-Hee $\mathrm{Oh}^{1}$, Young-Joo Lee ${ }^{1,2 *}$ \\ ${ }^{1}$ Division of Biopharmaceutics, College of Pharmacy, Kyung Hee University, Seoul, 130-701, Korea. \\ ${ }^{2}$ Department of Life and Nanopharmaceutical Sciences, Kyung Hee University, Seoul, 130-701, Korea.
}

Received, August 3, 2012; Accepted, September 28, 2012; Published, September 29, 2012.

\begin{abstract}
Purpose. Hyperlipidemia is associated with an increased risk of erectile dysfunction. In this study, we investigated the effects of hyperlipidemia on the pharmacokinetics of tadalafil, a novel therapeutic agent for erectile dysfunction, in rats with experimental hyperlipidemia. Methods. Tadalafil $(1 \mathrm{mg} / \mathrm{kg})$ was administered to control rats and rats with poloxamer-407-induced hyperlipidemia $(1 \mathrm{~g} / \mathrm{kg}$, i.p.). In addition, we performed in vitro studies to determine the hepatic metabolism in S9 fractions, intestinal absorption, and plasma protein binding. Results. Hyperlipidemia dramatically increased tadalafil's the total area under the plasma concentration-time curve from time 0 to infinity after intravenous (2.09-fold) and oral (11.9-fold) administration, and decreased total body clearance $(0.537$-fold) and apparent volume of distribution at the steady state $(0.438$ fold) after intravenous administration of tadalafil. Further, we observed decreased in vitro hepatic S9 metabolism, intestinal first-pass metabolism, and unbound fraction of tadalafil. Conclusions. The alterations in the pharmacokinetics of tadalafil observed in rats with poloxamer 407-induced hyperlipidemia may be attributable to a decrease in hepatic and intestinal metabolism and unbound fraction of tadalafil in the plasma. These findings have potential therapeutic implications for predicting the pharmacokinetic responses of humans to hyperlipidemia.
\end{abstract}

This article is open to POST-PUBLICATION REVIEW. Registered readers (see "For Readers") may comment by clicking on ABSTRACT on the issue's contents page.

\section{INTRODUCTION}

Tadalafil (Cialis ${ }^{\circledR}$ ), a novel therapeutic agent for erectile dysfunction, is a potent, reversible, competitive inhibitor of phosphodiesterase 5 (PDE-5), an enzyme that inactivates cyclic guanosine monophosphate (1). Tadalafil exerts its action by inhibiting PDE-5 in the corpus cavernosum of the penis and increasing the levels of intracellular cyclic guanosine monophosphate, thereby facilitating the relaxation of smooth muscles, which lead to penile erection $(2,3)$. An observational study showed that patients consuming tadalafil consistently had higher levels of therapeutic effectiveness and satisfaction than those of patients who consumed other PDE-5 inhibitors, sildenafil or vardenafil (4). Tadalafil has a longer duration of action (up to $36 \mathrm{~h}$ ) than sildenafil and vardenafil (approximately $4 \mathrm{~h}$ ) (5). Moreover, the absorption of sildenafil and vardenafil may be delayed in the presence of high-fat food; however, the rate and extent of tadalafil absorption are unaffected by a high-fat meal (5).The above advantages are responsible, in part, for the rapid increase in the market share of tadalafil for the treatment of erectile dysfunction (6).

Erectile dysfunction is also associated with hyperlipidemia (7). Hyperlipidemia is characterized by abnormally high levels of any or all lipids and/or lipoproteins in the blood. With high blood pressure, cigarette smoking, and diabetes, it is recognized as a risk factor for coronary heart disease, which is a leading cause of morbidity and mortality among adults in Europe and North America $(8,9)$. Of the 154 men who had symptoms of erectile dysfunction for at least 6 months, $74 \%$ had low-density lipoprotein cholesterol level of $>120 \mathrm{mg} / \mathrm{dL}$ (10). Erectile dysfunction in these patients is attributed to hypercholesterolemia-induced impairment of endothelium-dependent relaxation in the smooth muscle cells of the corpus cavernosum (11).

Corresponding Author: Prof. Young-Joo Lee; Division of Biopharmaceutics, College of Pharmacy; Kyung Hee University 1 Hoegi-dong Dongdaemun, Seoul, Korea; E-mail: yj_lee@khu.ac.kr 
Hyperlipidemia was shown to significantly alter pharmacokinetics of many drugs, including nifedipine (12), docetaxel (13), amiodarone (14), and cyclosporine A (15). The clearance of nifedipine was significantly lower in the hyperlipidemic rats due to the decrease in fraction unbound in plasma (12). For the docetaxel, the unbound fraction and intrinsic hepatic metabolism was significantly decreased possibily due to the lower expression of cytochrome P 450 (CYP) 3A (13). A substantial increase in the amiodarone plasma concentrations and decreases in the clearance, volume of distribution and unbound fraction were also noted in hyperlipidemic rats (14). The clearance of cyclosporine A was not changed in hyperlipidemic rats but the plasma unbound fraction, volume of distribution, and terminal half-life were each significantly decreased (15). Thus pharmacokinetics of tadalafil, which has a lipophilic property and is metabolized by CYP3A (16), might also be changed in the hyperlipidemic state.

The purpose of this study was to determine the changes in the pharmacokinetics of tadalafil in an animal model of hyperlipidemia induced by poloxamer 407 (P-407). P-407 can induce hyperlipidemia, which significantly increases the plasma cholesterol and triglyceride levels, in various animal models (17-19). We determined the plasma concentrations after intravenous and oral administration of tadalafil and the in vitro hepatic metabolism in S9 fractions, intestinal absorption, and plasma protein binding of tadalafil in control and hyperlipidemic rats.

\section{MATERIALS AND METHODS}

\section{Chemicals}

Acebutolol hydrochloride (internal standard for liquid chromatography and tandem mass spectrometry [LCMS/MS] of tadalafil), P-407, $\beta$-nicotinamide adenine dinucleotide phosphate, reduced tetra (cyclohexylammonium) salt (NADPH), Trizma base, and ethylenediaminetetraacetic acid (EDTA) dipotassium salt dehydrate were purchased from Sigma-Aldrich Corporation (St. Louis, MO, USA). All chemicals were of reagent grade or highperformance liquid chromatography (HPLC) grade.

\section{Animals}

Male Sprague-Dawley rats (250-280 g, Orient, Seoul, Korea) were used for the experiments. The rats were maintained at a temperature of $25^{\circ} \mathrm{C}$ and a $12-\mathrm{h}$ light/12-h dark cycle with free access to water and feed in a housing facility fully accredited by the Association for Assessment and Accreditation of Laboratory Animal Care International (Animal Center for Pharmaceutical Research, College of Pharmacy, Kyung Hee University, Seoul, Korea). All animal care and experimental procedures complied with the Guidelines of the Committee for Animal Care and Use of laboratory animals, College of Pharmacy, Kyung Hee University.

\section{Induction of hyperlipidemia in rats by $P-407$}

Rats were randomly assigned to receive either normal saline (control rats) or $1 \mathrm{~g} / \mathrm{kg} \mathrm{P}-407$ (hyperlipidemic rats) $(\mathrm{n}=13$, each). P-407 $(0.13 \mathrm{~g} / \mathrm{mL}$ solution in normal saline) was prepared by the cold method $24 \mathrm{~h}$ before use and kept at $2-5^{\circ} \mathrm{C}$ to maintain a liquid phase $(12,13)$. Approximately $36 \mathrm{~h}$ before the preparation of hepatic S9 fractions, the intestinal absorption study, and pharmacokinetic experiments, P-407 or normal saline was injected into the intraperitoneal cavity of each rat (14). Plasma concentrations of total cholesterol and triglycerides were determined using an automated clinical chemistry analyzer (Erba Diagnostics Mannheim $\mathrm{GmbH}$, Mannheim, Germany) and commercially available reagents (Stanbio Laboratory, TX, USA).

\section{Pharmacokinetics of tadalafil after intravenous and oral administration}

The rats were pretreated according to previously established procedures (13). One jugular vein (for drug administration in the intravenous study) and one carotid artery (for blood sampling) of each rat in both groups were cannulated using a polyethylene tube (Natsume, Tokyo, Japan); the rats were maintained under light ether anesthesia throughout the cannulation procedure. Both cannulas were exteriorized to the dorsal surface of the neck, where each cannula terminated in a long silastic tube (Dow Corning, Midland, MI, USA). Both silastic tubes were covered with a wire sheath to allow the rats to move freely. We used $0.3 \mathrm{~mL}$ of heparinized $0.9 \%$ $\mathrm{NaCl}$ injectable solution $(20 \mathrm{U} / \mathrm{mL})$ to flush the cannula to prevent blood clotting. Each rat was housed individually in a metabolic cage (Daejong Scientific Company, Seoul, Korea) and allows recovery from anesthesia for $4-5 \mathrm{~h}$ before the initiation of the experiment. Thus, all experiments were performed using fully conscious and freely mobile rats.

For the intravenous study, tadalafil (dissolved in polyethylene glycol 400:water $=1: 1$ ) was 
intravenously administered to rats at a dose of 1 $\mathrm{mg} / \mathrm{kg}(2 \mathrm{~mL} / \mathrm{kg})$ infused over $1 \mathrm{~min} 36 \mathrm{~h}$ after injecting normal saline (control rats, $\mathrm{n}=7$ ) or P-407 (hyperlipidemic rats, $\mathrm{n}=8$ ). Blood samples of approximately $220 \mu \mathrm{L}$ were collected from the carotid artery at intervals of $0,1,5,15,30,60,120$, 180 , and $240 \mathrm{~min}$ after the start of the intravenous infusion. We used $0.3 \mathrm{~mL}$ of heparinized $0.9 \% \mathrm{NaCl}$ injectable solution $(20 \mathrm{U} / \mathrm{mL})$ to flush each cannula immediately after each blood sampling to prevent blood clotting and to compensate for fluid loss. Blood samples were separated by centrifugation, and 100$\mu \mathrm{L}$ aliquots of the plasma were stored at $-80^{\circ} \mathrm{C}$ until tadalafil analysis.

For the oral administration study, rats were fasted overnight with free access to water. Tadalafil (dissolved in polyethylene glycol 400:water $=1: 1$ ) was administered orally via a feeding tube at a dose of $1 \mathrm{mg} / \mathrm{kg}(5 \mathrm{~mL} / \mathrm{kg})$ to both control $(n=6)$ and hyperlipidemic $(n=5)$ rats. Blood samples (approximately $220 \mu \mathrm{L}$ each) were collected from the carotid artery at $0,5,15,30,60,120,180,240,360$, and $480 \mathrm{~min}$ after the oral administration. The remaining procedures were performed as described above for the intravenous study.

\section{In vitro metabolism of tadalafil in hepatic S9 fractions}

The hepatic S9 fractions of the rat were prepared according to the previously reported protocol (13). Hepatic S9 homogenates (equivalent to $1 \mathrm{mg}$ protein) from control $(n=3)$ and hyperlipidemic $(n=3)$ rats were added to reaction mixtures containing $50 \mathrm{mM}$ Tris-HCl buffer (pH 7.4) and $1 \mathrm{mM}$ NADPH $(20-\mu \mathrm{L}$ aliquot of $50 \mathrm{mM}$ stock dissolved in $50 \mathrm{mM}$ Tris- $\mathrm{HCl}$ buffer) in a final volume of $1 \mathrm{~mL}$. To investigate the NADPH-independent degradation of tadalafil, negative control reaction mixtures containing only 50 $\mathrm{mM}$ Tris- $\mathrm{HCl}$ buffer (pH 7.4) and $1 \mathrm{mg}$ S9 homogenate protein in a final volume of $1 \mathrm{~mL}$ without NADPH were used. The reaction was initiated by adding $5 \mu \mathrm{L}$ of $0.2 \mathrm{mM}$ tadalafil solution in methanol (the final concentration of tadalafil was 1 $\mu \mathrm{M})$. Samples were incubated in a water bath at $37^{\circ} \mathrm{C}$ with shaking at a rate of 90 revolutions per min (rpm). A $100-\mu \mathrm{L}$ aliquot was collected at $0,15,30,60$, and $120 \mathrm{~min}$ after the start of the incubation and transferred to an Eppendorf tube containing $10 \mu \mathrm{L}$ of $100 \mathrm{ng} / \mathrm{mL}$ acebutolol in water : methanol=1:1 (internal standard) and $1 \mathrm{~mL}$ of tert-butyl methyl ether and vortex-mixed to terminate the reaction. The samples were then centrifuged at $15,000 \mathrm{rpm}$ for 10 min, and a 1-mL aliquot of the supernatant was evaporated to dryness using a Speed-Vac concentrator (CentraVac; Vision Scientific Co., Bucheon, Korea) and stored at $-80^{\circ} \mathrm{C}$ until analysis of tadalafil by LCMS/MS.

\section{Intestinal first-pass effect of tadalafil}

Rats were fasted overnight with free access to water. In addition, the portal veins of control $(n=4)$ and hyperlipidemic $(n=3)$ rats were cannulated (20) by the modified Suzuki method (21). An abdominal incision was made under tiletamine-zolazepam (25 $\mathrm{mg} / \mathrm{kg}$ each, intraperitoneal) anesthesia, the pyloric vein was isolated (to minimize impaired blood flowing in the portal vein), and the tapered end of a 27 -gauge needle bent at a $45^{\circ}$ angle was inserted into the vein. Bleeding was prevented by applying instant glue. A piece of long polyethylene tubing was attached to the other end of the needle and filled with heparinized saline $(20 \mathrm{IU} / \mathrm{mL})$ to prevent blood clotting.

Tadalafil (the same solution as that used in the intravenous study) was instilled into the duodenum of the rats, and blood samples of approximately $220 \mu \mathrm{L}$ were collected from the pyloric vein at 1 min after instillation of the drug. Blood samples were separated by centrifugation, and $100-\mu \mathrm{L}$ aliquots of the plasma were stored at $-80^{\circ} \mathrm{C}$ until tadalafil analysis.

\section{Measurement of plasma protein binding of tadalafil}

Protein binding of tadalafil in fresh plasma from control $(n=4)$ and hyperlipidemic $(n=4)$ rats was determined using the ultrafiltration method. The procedures were similar to a previously reported method (22). Plasma $(0.5 \mathrm{~mL})$ containing $1 \mu \mathrm{g} / \mathrm{mL}$ tadalafil was loaded into the sample reservoirs of Centrifree ${ }^{\circledR}$ ultrafiltration devices (regenerated cellulose 30000 MWCO, Millipore Co., MA, USA), and the ultrafiltrate, containing free tadalafil, was obtained by subjecting the system to low speed centrifugation $\left(1500 \times g, 30 \mathrm{~min}, 37^{\circ} \mathrm{C}\right)$. A $100-\mu \mathrm{L}$ aliquot of the ultrafiltrate was stored at $-80^{\circ} \mathrm{C}$ until used for tadalafil analysis. Protein-independent loss of tadalafil due to nonspecific binding to the ultrafiltration devices was evaluated using phosphate buffer ( $\mathrm{pH}$ 7.4) containing an equal concentration of tadalafil $(n=4)$.

\section{LC-MS/MS analysis}

The extraction efficiency of lipophilic drugs using organic solventsis altered by the presence of high 
concentrations of lipoprotein (23). Therefore, to determine the concentration of tadalafil in the plasma of hyperlipidemic rats using LC-MS/MS, we used drug-free plasma samples from rats treated in a manner similar to that of hyperlipidemic rats as a blank.

The concentrations of tadalafil in rat plasma and phosphate buffer samples were determined by LCMS/MS (Agilent Technologies 1200 series; Agilent, Santa Clara, CA, USA) coupled to a Waters Quattro Micro $^{\mathrm{TM}}$ API mass spectrometer (Waters, Milford, MA, USA) equipped with an electrospray ionization interface used to generate positive ions $[\mathrm{M}+\mathrm{H}]^{+}$. Plasma samples collected at $1 \mathrm{~min}$ after the start of the intravenous infusion in both groups were diluted 1:5 with drug-free plasma samples before sample preparation. We added $10-\mu \mathrm{L}$ aliquots of $100 \mathrm{ng} / \mathrm{mL}$ acebutolol in 50\% methanol (internal standard) and 1 $\mathrm{mL}$ of tert-butyl methyl ether to $100 \mu \mathrm{L}$ of plasma and buffer samples. Then, the mixture was vortexmixed for $5 \mathrm{~min}$ and centrifuged at 15,000 rpm for 10 min, and a $1-\mathrm{mL}$ aliquot of the supernatant was evaporated to dryness using a Speed-Vac concentrator (CentraVac; Vision Scientific Co.). The residue was reconstituted with $50 \mu \mathrm{L}$ of $50 \%$ methanol, and a 30 $\mu \mathrm{L}$ aliquot was directly injected onto a ThermoHypersil-Keystone $\mathrm{C}_{18}$ column $(50 \times 2.1 \mathrm{~mm}$ internal diameter [i.d.]; particle size, $5 \mu \mathrm{m}$; Thermo Fisher Scientific Inc., Waltham, MA, USA). The flow-rate of the mobile phase $0.1 \%$ formic acid:acetonitrile $(45: 55, \mathrm{v} / \mathrm{v})$ was $0.3 \mathrm{~mL} / \mathrm{min}$. Quantification was performed by multiple reaction monitoring (MRM) of the transitions of $\mathrm{m} / \mathrm{z} 390.25$ $\rightarrow 268.212$ for tadalafil and $\mathrm{m} / \mathrm{z} 337.2 \rightarrow 116$ for acebutolol. The optimal mass parameters obtained were as follows: capillary voltage, $3.7 \mathrm{kV}$; cone voltage, $27 \mathrm{~V}$ and $22 \mathrm{~V}$ for tadalafil and acebutolol, respectively; source temperature, $120^{\circ} \mathrm{C}$; and desolvation temperature, $400^{\circ} \mathrm{C}$. Nitrogen was used as the desolvation gas and cone gas with flow rates of $800 \mathrm{~L} / \mathrm{h}$ and $50 \mathrm{~L} / \mathrm{h}$, respectively. The collision energies were $18 \mathrm{eV}$ and $24 \mathrm{eV}$ for tadalafil and acebutolol, respectively. The analytical data were processed using MassLynx V 4.1 software (Waters).

\section{Data analysis}

For the ultrafiltration technique, the percentage binding was determined as follows:

$$
\left[\frac{(\mathrm{T}-\mathrm{F})}{\mathrm{T}}\right] \times 100
$$

where $F$ denotes the free-drug concentration measured in the ultrafiltrate and $\mathrm{T}$ is the total drug concentration introduced into the system (22).

We used standard methods (24) for noncompartmental analysis (WinNonlin Standard, version 3.1; Pharsight, Mountain View, CA, USA) to calculate the following pharmacokinetic parameters: the total area under the plasma concentration-time curve from time 0 to infinity $\left(\mathrm{AUC}_{0-\infty}\right)(25)$, total body clearance (CL), terminal half-life, mean residence time (MRT), and apparent volume of distribution at steady state $\left(V_{\mathrm{ss}}\right)$. The peak plasma concentration $\left(C_{\max }\right)$ and time to reach $C_{\max }\left(T_{\max }\right)$ were determined directly from the experimental data.

Statistical analysis was performed using an unpaired Student's $t$-test with a two-tailed distribution. A $p$-value less than 0.05 was considered statistically significant. All data are expressed as mean \pm standard deviation (SD).

\section{RESULTS}

\section{LC-MS/MS analysis of tadalafil}

No impurities or interfering peaks from endogenous substances were observed at any elution time for tadalafil or acebutolol. The LC-MS/MS method was linear $\left(r^{2}>0.99\right)$ over the tadalafil concentration ranges of 2-2000 and $2-1000 \mathrm{ng} / \mathrm{mL}$ for plasma and buffer samples, respectively, when evaluated by the least-squares linear regression. The lower limit of quantification (LLOQ) was set at the lowest standard concentration $(2 \mathrm{ng} / \mathrm{mL})$ in the calibration curve. The accuracy (measured by relative \% error) and intraday precision (evaluated as the relative standard deviation of the mean expressed as a percent; coefficient of variation) of this method indicated that all relative errors and coefficients of variation at each concentration level were below $15 \%$.

\section{Pharmacokinetics of tadalafil after intravenous and oral administration}

The plasma concentration-time profiles of tadalafil after intravenous administration to control and hyperlipidemic rats are shown in Fig. 1 (A), and the relevant pharmacokinetic parameters are listed in Table 1. Compared to control rats, hyperlipidemic rats showed a significant increase in $\mathrm{AUC}_{0-\infty}$ (2.09fold) and significant decreases in CL and $V_{\mathrm{ss}}(0.537$ and 0.438 -fold, respectively) after intravenous administration of tadalafil. The terminal half-life and MRT were comparable between the hyperlipidemic and control rats. 
The plasma concentrations-time profiles after oral administration of tadalafil to control and hyperlipidemic rats are shown in Fig. 1 (B), and the relevant pharmacokinetic parameters are listed in Table 1. Compared to control rats, hyperlipidemic rats showed significant increases in $\mathrm{AUC}_{0-\infty}$ and $C_{\max }$ (11.9- and 5.91-folds, respectively) and a significant decrease in the apparent CL value (0.0711-fold) after oral administration of tadalafil. The absolute oral bioavailability values of tadalafil were significantly greater (5.61-fold) in the hyperlipidemic rats compared to control rats.

\section{In vitro metabolism of tadalafil in hepatic S9 fractions}

The percentages of tadalafil remaining after $0,15,30$, 60 , and $120 \mathrm{~min}$ of incubation with the S9 fractions of the livers of control and hyperlipidemic rats are shown in Figure 2. After 120min of incubation, 64.9\% of the tadalafil remained in the hepatic S9 fraction of the hyperlipidemic rats compared to $39.2 \%$, which remained in the hepatic S9 fraction of control rats. When incubated without NADPH, no metabolism or degradation of tadalafil was observed in the hepatic S9 fractions of either the control or the hyperlipidemic rats.

(A)

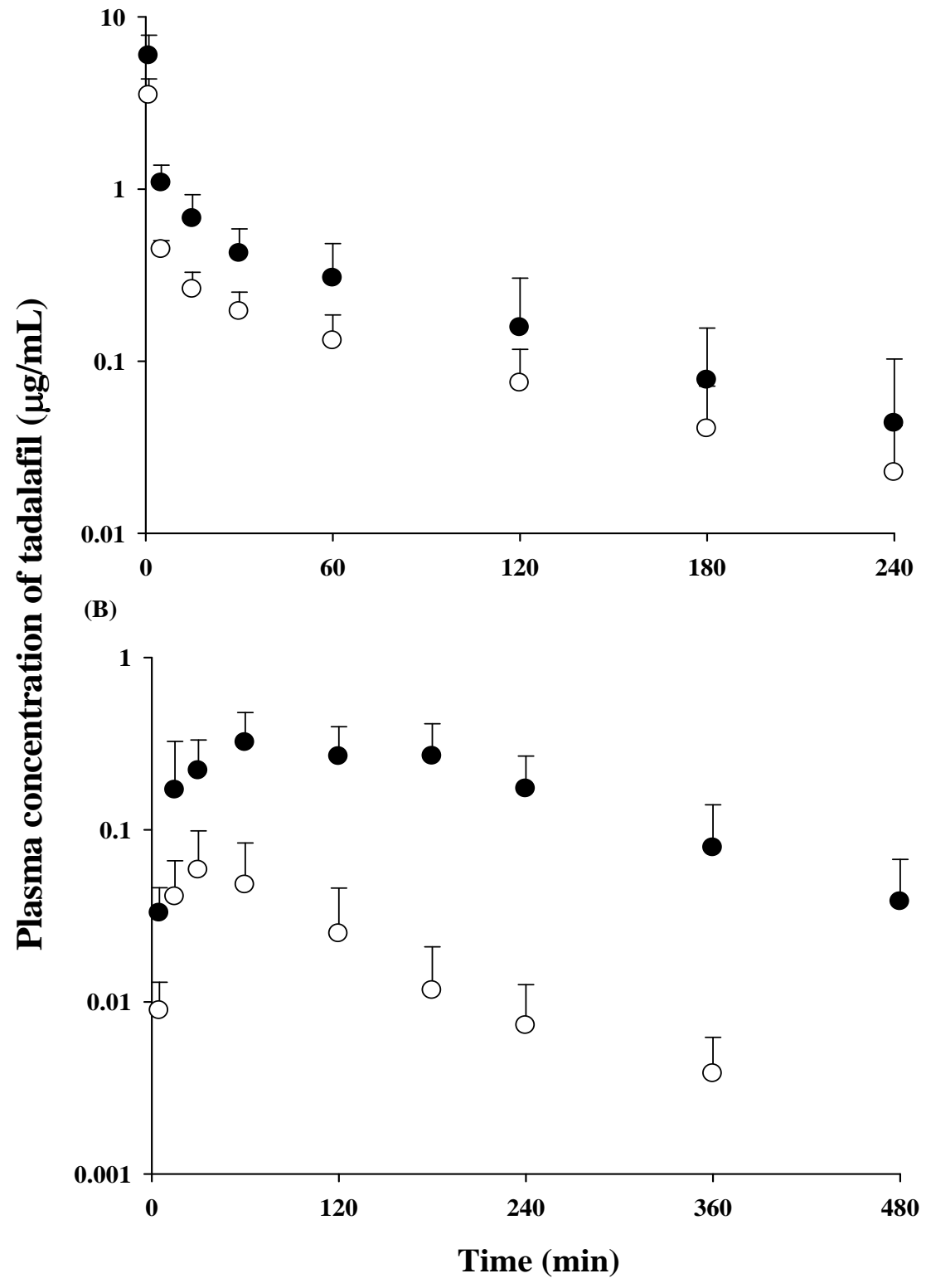

Figure 1. Mean plasma concentration-time curves of tadalafil after intravenous (A) and oral (B) administration at a dose of $1 \mathrm{mg} / \mathrm{kg}$ in control (०) and hyperlipidemic $(\bullet)$ rats. Bars represent SD. 
Table 1. Pharmacokinetic parameters of tadalafil after intravenous and oral administration at a dose of $1 \mathrm{mg} / \mathrm{kg}$ to the control (CON) and hyperlipidemic (HL) rats. Data are represented as mean \pm SD. ${ }^{*} p<0.05,{ }^{* * *} p<0.01$, and ${ }^{* * *} p<0.001$ vs. control rats (Student's $t$-test).

\begin{tabular}{|c|c|c|c|c|c|c|c|c|c|c|c|c|}
\hline \multirow{3}{*}{$\begin{array}{l}\text { Parameter } \\
\text { Total Body Weight (g) }\end{array}$} & \multicolumn{6}{|c|}{ Intravenous administration } & \multicolumn{6}{|c|}{ Oral administration } \\
\hline & \multicolumn{3}{|c|}{$\mathrm{CON}(n=7)$} & \multicolumn{3}{|c|}{$\operatorname{HL}(n=8)$} & \multicolumn{3}{|c|}{$\mathrm{CON}(n=6)$} & \multicolumn{3}{|c|}{$\operatorname{HL}(n=5)$} \\
\hline & 302 & \pm & 8 & 306 & \pm & 4 & 281 & \pm & 6 & 279 & \pm & 5 \\
\hline Total cholesterol (mg/dL) & 82.5 & \pm & 13.7 & 780 & \pm & $199^{* * *}$ & 77.8 & \pm & 12.5 & 835 & \pm & $90^{* * *}$ \\
\hline Triglyceride (mg/dL) & 93.6 & \pm & 49.6 & 2720 & \pm & $600^{* * *}$ & 87.3 & \pm & 39.5 & 2930 & \pm & $0^{* * *}$ \\
\hline $\operatorname{AUC}_{0-\infty}(\mu \mathrm{g} / \mathrm{mL} \cdot \min )$ & 35.5 & \pm & 12.9 & 74.1 & \pm & $38.8^{*}$ & 7.38 & \pm & 4.59 & 87.8 & \pm & $43.9^{* *}$ \\
\hline Terminal half-life(min) & 61.2 & \pm & 21.8 & 52.9 & \pm & 20.4 & & & & & & \\
\hline MRT (min) & 64.1 & \pm & 28.4 & 56.6 & \pm & 32.7 & & & & & & \\
\hline$C_{\max }(\mu \mathrm{g} / \mathrm{mL})$ & & & & & & & 0.0597 & \pm & 0.0406 & 0.353 & \pm & $0.106^{* * *}$ \\
\hline$T_{\max }(\min )$ & & & & & & & 32.5 & \pm & 14.7 & 87.0 & \pm & 64.0 \\
\hline $\mathrm{CL}(\mathrm{mL} / \mathrm{min} / \mathrm{kg})$ & 31.5 & \pm & 10.9 & 16.9 & \pm & $8.4^{*}$ & 204 & \pm & 148 & 14.5 & \pm & $8.7^{*}$ \\
\hline$V_{\mathrm{ss}}(\mathrm{mL} / \mathrm{kg})$ & 1780 & \pm & 370 & 779 & \pm & $213^{* * *}$ & & & & & & \\
\hline$F(\%)$ & & & & & & & & 20. & & & & \\
\hline
\end{tabular}

\section{Intestinal first-pass effect of tadalafil}

The concentrations of tadalafil in the pyloric vein at 1 min after instillation into the duodenum were $68.7 \pm$ 6.3 and $182 \pm 4 \mu \mathrm{g} / \mathrm{mL}$ in the control and hyperlipidemic rats, respectively $(p<0.001)$.

\section{Plasma protein binding of tadalafil}

The plasma protein binding values of tadalafil were $91.1 \pm 0.7 \%$ and $98.4 \pm 0.1 \%$ for control $(n=4)$ and hyperlipidemic $(n=4)$ rats, respectively; this difference was statistically significant $(p<0.001$, Figure 3). When phosphate buffer ( $\mathrm{pH}$ 7.4) containing tadalafil was subjected to ultrafiltration, the mean recovery was $101 \pm 6 \%(n=4)$. This indicates negligible protein-independent loss of tadalafil due to nonspecific binding to the ultrafiltration apparatus.

\section{DISCUSSION}

Induction of hyperlipidemia by $\mathrm{P}-407$ in rats is a well-established animal model of hyperlipidemia and is beneficial for the evaluation of newer classes of antihyperlipidemic agents (26). This model is widely used because of ease of use, reproducibility, and lack of undesirable pathological conditions (19). Induction of hyperlipidemia in rats by $\mathrm{P}-407$ is associated with marked alterations in the activities of plasma lecithin cholesterol acyltransferase, cholesteryl ester transfer protein, hepatic lipase, and lipoprotein lipase, which are responsible for lipid metabolism (27). In our study, induction of hyperlipidemia in rats by $\mathrm{P}-407$ was evident from the significantly high levels of total plasma cholesterol and triglyceride (Table 1). 
(A)

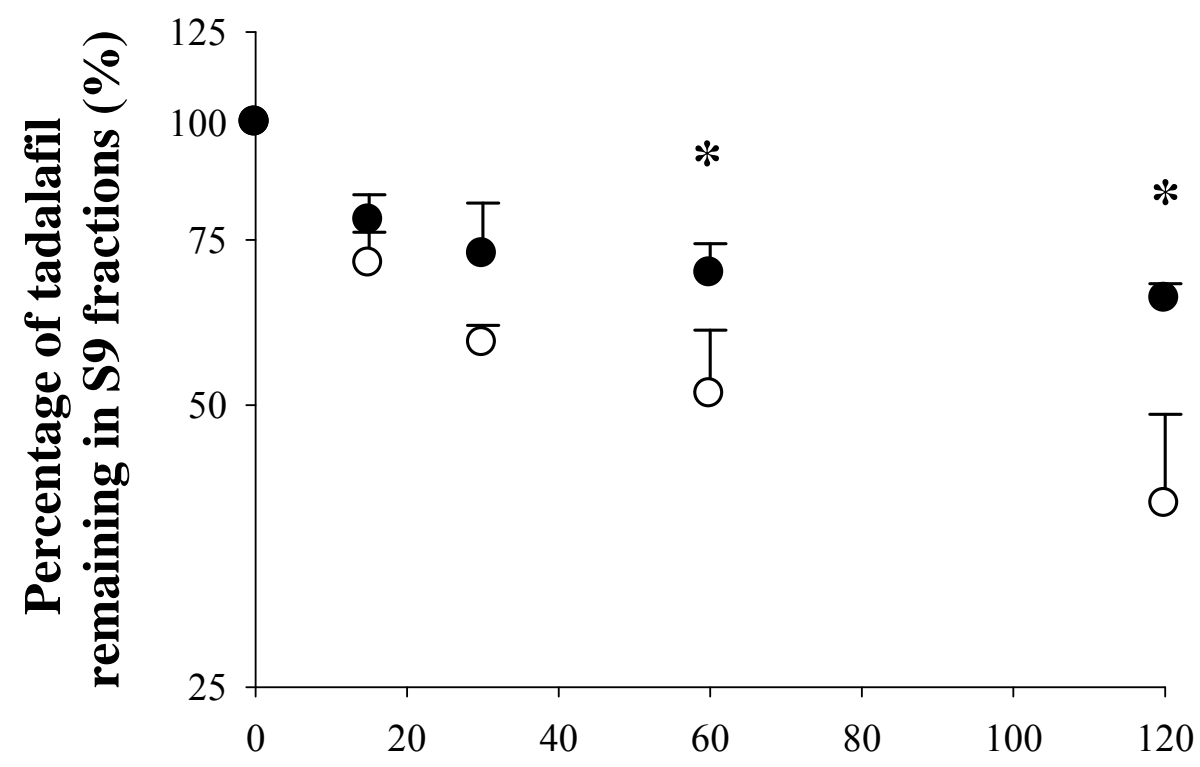

(B)

Time (min)

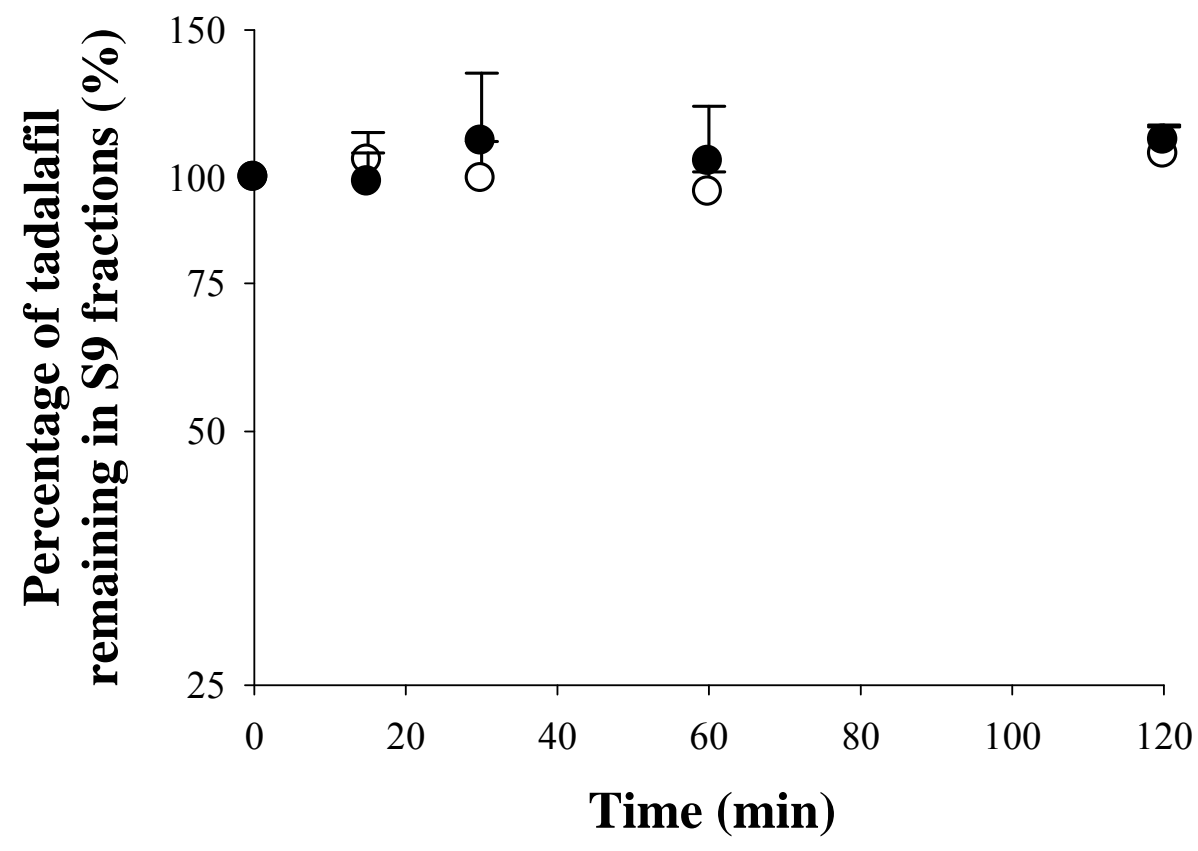

Figure 2. Percentages of tadalafil remaining after incubation for $0,15,30,60$, and 120 min with hepatic $\mathrm{S} 9$ fractions from control (O) and hyperlipidemic $(\bullet)$ rats with (A) and without (B) NADPH. Bars represent SD. ${ }^{*} p<0.05$ vs. control rats (Student's t-test) 


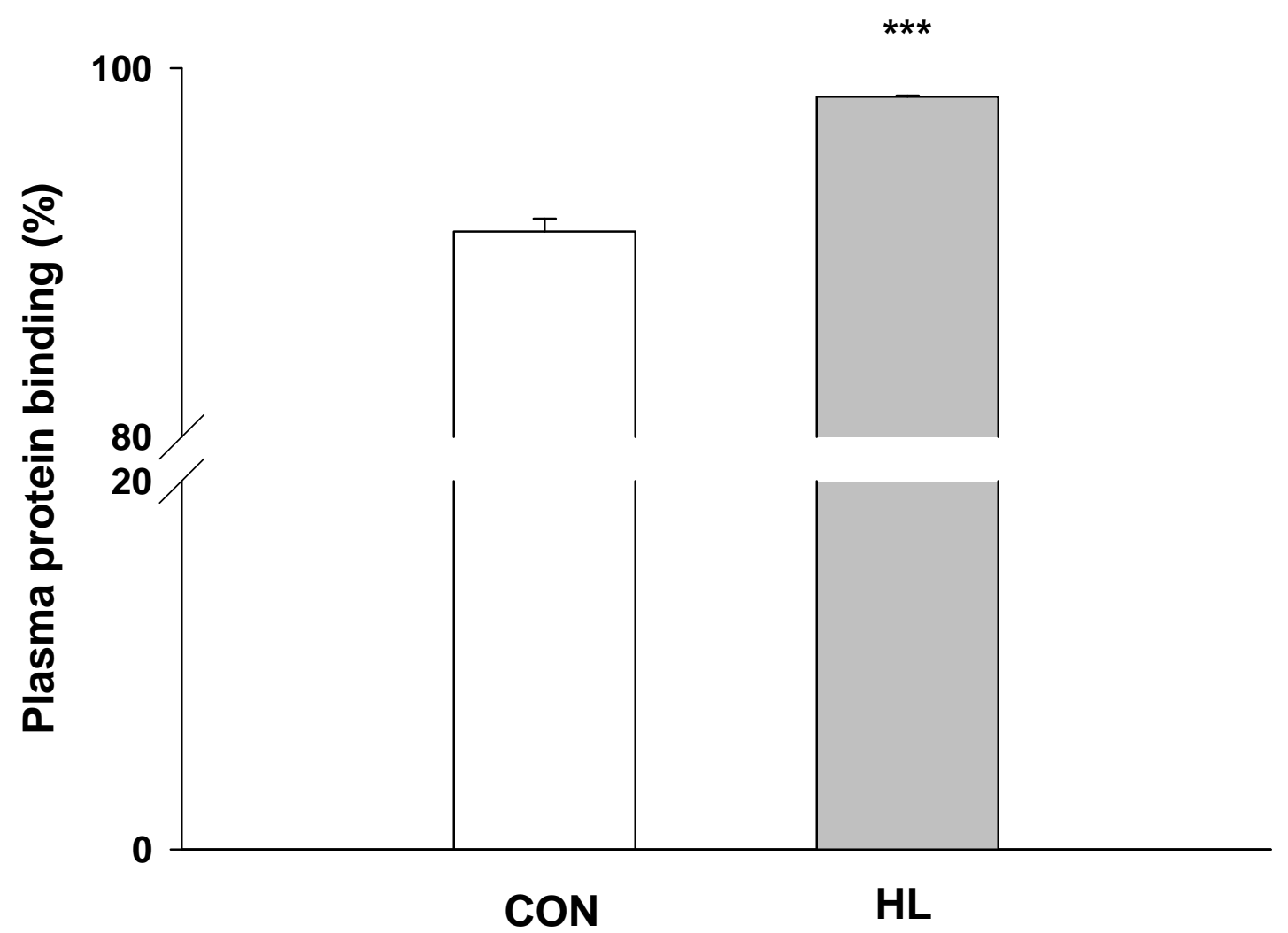

Figure 3. Plasma protein binding values of tadalafil to proteins in fresh plasma from control (CON, white column, $n=4$ ) and hyperlipidemic (HL, gray column, $n=4$ ) rats determined by the ultrafiltration method. Bars represent SD. ${ }^{* * *} p<0.001$ vs. control rats (Student's $t$-test)

The significant decrease in the values of $\mathrm{CL}$, and consequently the increase in $\mathrm{AUC}_{0-\infty}$ of intravenously administered tadalafil in hyperlipidemic rats (Table 1) could be because of the lesser degree of hepatic metabolism in these rats than in control rats. Because tadalafil is a drug with a low hepatic extraction ratio (28), its disposition may be susceptible to changes in intrinsic metabolic clearance (29). The significantly lesser degree of hepatic metabolism in the hyperlipidemic rats was supported by a significantly lower degree metabolism of tadalafil by the hepatic S9 fractions in the hyperlipidemic rats than in control rats (Figure. 2).

It has been previously demonstrated that the total cytochrome P 450 (CYP) content was significantly lower in the microsomal protein of the liver of hyperlipidemic rats $(0.407 \pm 0.0883)$ than of control rats $(0.788 \pm 0.183 \mathrm{nmol} / \mathrm{mg}$ protein) (30). Shayeganpour et al. (30) showed significant decreases in the protein expressions of CYP2C11,
CYP3A1, and CYP3A2 (39.6\%, 51.9\%, and 38.4\%, respectively) in the hepatic microsomes of hyperlipidemic rats compared to control rats. In humans, tadalafil clearance is predominately via hepatic metabolism by CYP3A to a catechol that undergoes methylation and is extensively conjugated to form the major circulating metabolite, a methylcatechol glucuronide (16). Although the degree of contribution of CYP3A to the metabolism of tadalafil in rats has not been identified thus far, it could be inferred that the lower clearance of tadalafil in the hyperlipidemic rats resulted from the decreased expression of hepatic CYP3A1/2 when the $73 \%$ homology between human CYP3A4 and rat CYP3A1 protein was considered (31). Results similar to ours in the hyperlipidemic rats have been reported for docetaxel (13), nifedipine (12), and atazanavir (32).

Lipoproteins interact with a number of lipophilic drugs, and hyperlipidemia increases the binding capacity of the lipoproteins (33). Actually, in the P- 
407-induced hyperlipidemic rats, the value of protein binding increased and thus the volume of distribution was less $(13,14)$. In our study, the unbound fraction of tadalafil was significantly lower (0.175-fold, Figure 3 ) in the plasma of the hyperlipidemic rats compared with that of the controls. The $V_{\text {ss }}$ of tadalafil was significantly smaller $(0.438$-fold $)$ in hyperlipidemic rats than in the control rats (Table 1 ), in line with the decrease in the unbound fraction. Further, this may have been responsible for the lower hepatic clearance of tadalafil.

After oral administration, the $\mathrm{AUC}_{0-\infty}$ of tadalafil in hyperlipidemic rats was 11.9 times higher than that of the control rats. This value was relatively higher (2.09-fold) than that after intravenous administration (Table 1). Therefore, the increase in the $\mathrm{AUC}_{0-\infty}$ value in hyperlipidemic rats after oral administration could not be explained by decreased hepatic clearance of tadalafil alone. To identify the intestinal first-pass effect of tadalafil in rats, the drug was directly spiked in the duodenum, and blood was collected from the pyloric vein in a very short time $(1 \mathrm{~min})$, which minimized the effects of systemic (hepatic) metabolism. The concentration of tadalafil in the portal vein was significantly higher (2.65-fold) in the hyperlipidemic rats than in the control rats, which suggested a lower intestinal first-pass effect in the hyperlipidemic rats. Thus, decreased intestinal metabolism combined with decreased hepatic metabolism of tadalafil after oral administration may be the cause of the greater $\mathrm{AUC}_{0-\infty}$ value in hyperlipidemic rats.

Although not reported previously, the possibility that P-407 itself might affect the liver or intestinal metabolizing enzymes should not be excluded. However, in this study, P-407 was administered intraperitoneally and tadalafil was administered 36 hours after injection of P-407. Therefore, it may be possible that only low concentrations of P-407 were present in the liver and intestine, perhaps below that needed to inhibit metabolizing enzyme. In our preliminary study, an induction effect of P-407 on the liver enzymes was not observed (data not shown). This could be one of the reasons for using P-407induced hyperlipidemia rats as an appropriate model.

There are some differences in the pharmacokinetic parameters of tadalafil between rats and humans, especially considerably longer half-life in humans (5). This discrepancy may be attributable to the interspecies difference. It is well known that metabolic rate of small animal such as rat is much faster than that of human (34). As we cannot know the exact mechanism of this interspecies difference, we cannot exclude the possibility that this interspecies difference may affect the interpretation of clinical impact of this modified pharmacokinetics of tadalafil in rats. Thus, caution should be exercised when extrapolating results from experimental animal models to humans. This is especially true for the present model because of the extremely high total cholesterol and triglyceride levels in the P-407treated rats (Table 1) compared with hyperlipidemic human clinical patients. Nevertheless, this model qualitatively represents the features of human hyperlipidemia.

In conclusion, the $\mathrm{AUC}_{0-\infty}$ value of tadalafil after intravenous administration to P-407-induced hyperlipidemic rats significantly increased because of decreases in intrinsic hepatic metabolism of tadalafil. In addition, the AUC value was significantly greater in the hyperlipidemic group after oral administration, possibly because of the combined effects of decreased hepatic and intestinal metabolism and unbound fraction of tadalafil in the plasma. These findings have potential therapeutic implications for the prediction of changes in the pharmacokinetics of drugs due to hyperlipidemia in humans.

\section{ACKNOWLEDGEMENTS}

This work was supported by a grant from the Kyung Hee University in 2011. (KHU-20110094)

\section{REFERENCES}

1. Murad F. Cyclic GMP: synthesis, metabolism, and function. Introduction and some historical comments. Adv Pharmacol, 1994;26:1-5.

2. Lue TF. Erectile dysfunction. N Engl J Med, 2000;342(24):1802-13.

3. Thompson WJ. Cyclic nucleotide phosphodiesterases: pharmacology, biochemistry and function. Pharmacol Ther, 1991;51(1):13-33.

4. Martin-Morales A, Haro JM, Beardsworth A, Bertsch J, Kontodimas S. Therapeutic effectiveness and patient satisfaction after 6 months of treatment with tadalafil, sildenafil, and vardenafil: results from the erectile dysfunction observational study (EDOS). Eur Urol, 2007;51(2):541-50.

5. Seftel AD. Phosphodiesterase type 5 inhibitor differentiation based on selectivity, pharmacokinetic, and efficacy profiles. Clin Cardiol, 2004;27(4 Suppl 1):I14-9.

6. Cleves AE, Jain AN. Effects of inductive bias on computational evaluations of ligand-based modeling and on drug discovery. J Comput Aided Mol Des, 
2008;22(3-4):147-59.

7. Roumeguere T, Wespes E, Carpentier Y, Hoffmann P, Schulman CC. Erectile dysfunction is associated with a high prevalence of hyperlipidemia and coronary heart disease risk. Eur Urol, 2003;44(3):355-9.

8. McGovern PG, Pankow JS, Shahar E, Doliszny KM, Folsom AR, Blackburn H, Luepker RV. Recent trends in acute coronary heart disease--mortality, morbidity, medical care, and risk factors. The Minnesota Heart Survey Investigators. N Engl J Med, 1996;334(14):884-90.

9. Gordon T, Kannel WB. Multiple risk functions for predicting coronary heart disease: the concept, accuracy, and application. Am Heart J, 1982;103(6):1031-9.

10. Walczak MK, Lokhandwala N, Hodge MB, Guay AT. Prevalence of cardiovascular risk factors in erectile dysfunction. J Gend Specif Med, 2002;5(6):19-24.

11. Azadzoi KM, Saenz de Tejada I. Hypercholesterolemia impairs endothelium-dependent relaxation of rabbit corpus cavernosum smooth muscle. J Urol, 1991;146(1):238-40.

12. Eliot LA, Foster RT, Jamali F. Effects of hyperlipidemia on the pharmacokinetics of nifedipine in the rat. Pharm Res, 1999;16(2):309-13.

13. Lee $\mathrm{JH}, \mathrm{Oh} \mathrm{JH}$, Lee YJ. Effects of experimental hyperlipidaemia on the pharmacokinetics of docetaxel in rats. Xenobiotica, 2011;41(9):797-804.

14. Shayeganpour A, Jun AS, Brocks DR. Pharmacokinetics of Amiodarone in hyperlipidemic and simulated high fat-meal rat models. Biopharm Drug Dispos, 2005;26(6):249-57.

15. Brocks DR, Ala S, Aliabadi HM. The effect of increased lipoprotein levels on the pharmacokinetics of cyclosporine A in the laboratory rat. Biopharm Drug Dispos, 2006;27(1):7-16.

16. Ring BJ, Gillespie JS, Mullen JH, Wrighton SA. Identification of the human cytochrome P450 (CYP) responsible for the formation of the major oxidative metabolite of tadalafil (Cialis (R)). Drug Metab Rev, 2004;36:70.

17. Palmer WK, Emeson EE, Johnston TP. Poloxamer 407-induced atherogenesis in the C57BL/6 mouse. Atherosclerosis, 1998;136(1):115-23.

18. Johnston TP, Palmer WK. Mechanism of poloxamer 407-induced hypertriglyceridemia in the rat. Biochem Pharmacol, 1993;46(6):1037-42.

19. Wout ZG, Pec EA, Maggiore JA, Williams RH, Palicharla P, Johnston TP. Poloxamer 407-mediated changes in plasma cholesterol and triglycerides following intraperitoneal injection to rats. J Parenter Sci Technol, 1992;46(6):192-200.

20. Kim EJ, Bae SK, Kim HJ, Kim YG, Kim SO, Lee DH, Lim H, Lee MG. Dose-independent pharmacokinetics of a candidate for diabetic neuropathy, SR-4668, after intravenous and oral administration to rats: Intestinal first-pass effect. J Pharm Sci, 2003;92(5):1112-24.

21. Xu ZX, Tang JP, Badr M, Melethil S. Kinetics of aluminum in rats. III: Effect of route of administration. J Pharm Sci, 1992;81(2):160-3.

22. Barre J, Chamouard JM, Houin G, Tillement JP. Equilibrium dialysis, ultrafiltration, and ultracentrifugation compared for determining the plasma-protein-binding characteristics of valproic acid. Clin Chem, 1985;31(1):60-4.

23. Porter CJ, Caliph SM, Charman WN. Differences in pre- and post-prandial plasma lipid profiles affect the extraction efficiency of a model highly lipophilic drug from beagle dog plasma. J Pharm Biomed Anal, 1997;16(1):175-80.

24. Gibaldi M, Perrier D. Pharmacokinetics.2nd ed., Marcel Dekker (eds), New York, NY, 1982.

25. Chiou WL. Critical evaluation of the potential error in pharmacokinetic studies of using the linear trapezoidal rule method for the calculation of the area under the plasma level--time curve. J Pharmacokinet Biopharm, 1978;6(6):539-46.

26. Johnston TP. The P-407-induced murine model of dose-controlled hyperlipidemia and atherosclerosis: a review of findings to date. J Cardiovasc Pharmacol, 2004;43(4):595-606.

27. Wasan KM, Subramanian R, Kwong M, Goldberg IJ, Wright T, Johnston TP. Poloxamer 407-mediated alterations in the activities of enzymes regulating lipid metabolism in rats. J Pharm Pharm Sci, 2003;6(2):189-97.

28. Forgue ST, Patterson BE, Bedding AW, Payne CD, Phillips DL, Wrishko RE, Mitchell MI. Tadalafil pharmacokinetics in healthy subjects. $\mathrm{Br} \mathrm{J}$ Clin Pharmacol, 2006;61(3):280-8.

29. Wilkinson GR, Shand DG. Commentary: a physiological approach to hepatic drug clearance. Clin Pharmacol Ther, 1975;18(4):377-90.

30. Shayeganpour A, Korashy H, Patel JP, El-Kadi AO, Brocks DR. The impact of experimental hyperlipidemia on the distribution and metabolism of amiodarone in rat. Int J Pharm, 2008;361(1-2):78-86.

31. Lewis DFV. Substrate specificity and metabolism. Cytochromes P450 Structure, Function and Mechanism. Bristol, PA: Taylor \& Francis; 1996. p 123.

32. Fukushima K, Shibata M, Mizuhara K, Aoyama H, Uchisako R, Kobuchi S, Sugioka N, Takada K. Effect of serum lipids on the pharmacokinetics of atazanavir in hyperlipidemic rats. Biomed Pharmacother, 2009;63(9):635-42.

33. Kwong TC, Sparks JD, Sparks CE. Lipoprotein and protein binding of the calcium channel blocker diltiazem. Proc Soc Exp Biol Med, 1985;178(2):313-6.

34. Kleiber M. Body size and metabolic rate. Physiol Rev, 1947;27(4):511-41. 